\title{
Ecophysiology of halophytes: questions and challenges
}

\author{
Sergey Shabala \\ School of Agricultural Science, University of Tasmania, Private bag 54, \\ Hobart, Tas 7001 \\ e-mail: Sergey.Shabala@utas.edu.au
}

\begin{abstract}
Summary. Halophytes are able to tolerate and even benefit from salt concentrations that kill most other plant species and, at the very least, may provide genes that allow transgenic conference of salinity tolerance to crops. In addition, some halophytes have already been tested as vegetable, forage and oilseed crops. However, physiological mechanisms behind the remarkable ability of halophytes to live in highly saline environment are not fully understood. This talk highlights some unanswered questions and challenges in understanding halophyte physiology, with a specific emphasis on complexity of plant-environmental interaction.
\end{abstract}

Key words: osmotic adjustment, salinity, oxidative stress, sodium, potassium, stomata.

\section{Halophyte anatomy: is anything special about it?}

Halophytes do not possess any specific 'anatomical hallmarks' that confer this remarkable salinity tolerance, and that will make them strikingly different from glycophytes. However, although the particular structures such as glands or bladders, and tissue succulence are not specifically unique to halophytes, it is apparent that the way the halophytes utilize these features is. Halophytism appears to be an integration of a large number of adaptive (mainly physiological) mechanisms rather than a distinct morphological or anatomical feature. The most important of these are; root suberisation, earlier vacuolisation of cells of the root apex, leaf and root succulency, and presents of salt bladders/glands. The latter two features are essential for the storage of (potentially toxic) excessive $\mathrm{Na}^{+}$. Salt bladders may also play an important role as a secondary epidermis to reduce water loss and prevent excessive UV damage. Up to know, mechanisms of ion loading into bladder cells remain an area of conjecture. In this talk, I will summarise the current knowledge on pathways for $\mathrm{Na}^{+}$transport into the bladder cells and some of the work undertaken in our laboratory to address this issue. Also, at least $50 \%$ of halophytes do not utilize glands or external bladders to modulate their tissue ion concentration. This poses the question; how do gland-less halophytes control $\mathrm{Na}^{+}$transport and sequestration, and is there any difference in the functional expression of $\mathrm{Na}^{+}$transporters between halophyte species with and without salt glands?

\section{Osmotically adjustment: organic or inorganic?}

Plants respond to hyperosmotic stress by an increase in intracellular osmolality, adjusting their cell turgor to altered growth conditions. This can be achieved either by increased uptake or de novo synthesis of a variety of organic osmolytes (so-called "compatible solutes"), or by controlling fluxes of ions across cellular membranes. The relative contribution of each of these mechanisms has been debated in literature for many years and remains unresolved. It is widely accepted that cell turgor in halophytes is maintained 
by storage of $\mathrm{Na}^{+}$and $\mathrm{Cl}^{-}$in vacuoles, with the solute potential of the cytosol adjusted by accumulation of $\mathrm{K}^{+}$and organic solutes. This view may be a gross oversimplification. In my talk, I present some of our recent data quantifying amounts of inorganic and organic osmolytes in halophytes species and show that accumulation patterns differed dramatically as a function of leaf age. Much less $\mathrm{Na}^{+}$being accumulated in young developing leaves, where inorganic ions contributed to only ca $50 \%$ of cell osmolality; the rest was attributed to de novo synthesis and accumulation of organic osmolytes. It is argued that the latter process plays a dual role providing, in addition to osmotic adjustment, protection of photosynthetic machinery against oxidative stress in (less vacuolated and less photosynthetically competent) developing leaves. I also discuss the energy cost for two different strategies of osmotic adjustment and implications of these findings for crop breeding for salinity tolerance.

\section{3. $\mathrm{Na}^{+}$sequestration: where and how?}

Efficient $\mathrm{Na}^{+}$sequestration is another hallmark of halophyte species. Given the lack of salt bladders in ca $50 \%$ of halophyte species, control of $\mathrm{Na}^{+}$loading into vacuole is absolutely essential. $\mathrm{Na}^{+}$sequestration is achieved via tonoplast $\mathrm{Na}^{+} / \mathrm{H}^{+}$antiporters. Tonoplast antiporters are constitutive in halophytes, whereas they must be activated by $\mathrm{NaCl}$ in salt-tolerant glycophytes, while in salt-sensitive plants their expression levels are extremely low and not salt-inducible. However, while NHX1 activity was detected in the plasma membrane fractions of Mesembryanthemum roots, tonoplast $\mathrm{Na}^{+} / \mathrm{H}^{+}$exchanger activity was not observed in either control or $\mathrm{NaCl}$-treated roots. Hence; the relation between salinity and a tonoplast $\mathrm{Na}^{+} / \mathrm{H}^{+}$exchanger in halophyte root activity is not as straightforward as one would expect. Also given the 4 to 5 fold concentration gradient between the vacuole and the cytosol, $\mathrm{Na}^{+}$ may easily leak back, unless some efficient mechanisms are in place to prevent this process. Specific details remain obscure. In this talk, I summarise our current knowledge on this subject and highlight the requirement to explicitly characterise the functional properties and gating modes of vacuolar channels mediating $\mathrm{Na}^{+}$and $\mathrm{K}^{+}$transport across the tonoplast in different halophyte families.

\section{Transpiration control: Are having fewer stomata better?}

While most authors agree that halophytes generally show a decline in transpiration rate at salinities above optimal, some exceptions are reported. The observed reduction in stomatal conductance in halophyte leaves is assumed to be important for improvements in water use efficiency and may originate from both morphological and physiological adaptive responses to salinity. A comparison between glycophyte species Arabidopsis thaliana and its halophitic relative Thellungiella halophila revealed about two-fold higher stomata density in Thellungiella leaves. At the same time, some other papers report reduction in stomatal density in plants grown at high salinity. In this talk, I explore the physiological relevance of this phenomenon referring to our quinoa (Chenopodium quinoa) data. I argue that the observed reduction in stomatal density may represent a fundamental mechanism by which plants optimise water use efficiency (WUE) under saline conditions. I also discuss advantages and a danger of halophytes' ability of substituting $\mathrm{K}^{+}$by $\mathrm{Na}^{+}$in the stomatal mechanism.

\section{ROS: friends of foes?}

Increased reactive oxygen species (ROS) production has been reported in response to a variety of abiotic and biotic stresses, including salinity. It has been repeatedly stated that the capacity to limit oxidative damage is important for halophytes' salt tolerance, and that halophytes possess higher oxidative stress tolerance than glycophytes. As a rule of thumb, activity of all major antioxidant systems increases noticeably under salt stress. However, for some species, no significant changes, or even a decrease in activity of some antioxidant enzymes have been reported. In this talk, I discuss this (apparently "odd") behaviour in the context of reactive oxygen species being important second messengers and, hence, a plant's requirement to maintain a fine balance between ROS production and scavenging by antioxidants. I also link intracellular ion homeostasis with Programmed Cell Death (PCD), and discuss how both these processes are affected by other environmental constraints such as drought, waterlogging, and extreme temperatures. 